\title{
EMBEDDING PERMUTATION GROUPS INTO WREATH PRODUCTS IN PRODUCT ACTION
}

\author{
CHERYL E. PRAEGER ${ }^{\bowtie}$ and CSABA SCHNEIDER
}

(Received 28 April 2011; accepted 16 January 2012)

Communicated by I. E. Shparlinski

This paper is dedicated to the memory of Alf van der Poorten

\begin{abstract}
We consider the wreath product of two permutation groups $G \leq \operatorname{Sym} \Gamma$ and $H \leq \operatorname{Sym} \Delta$ as a permutation group acting on the set $\Pi$ of functions from $\Delta$ to $\Gamma$. Such groups play an important role in the O'Nan-Scott theory of permutation groups and they also arise as automorphism groups of graph products and codes. Let $X$ be a subgroup of $\operatorname{Sym} \Gamma$ ? $\operatorname{Sym} \Delta$. Our main result is that, in a suitable conjugate of $X$, the subgroup of Sym $\Gamma$ induced by a stabiliser of a coordinate $\delta \in \Delta$ only depends on the orbit of $\delta$ under the induced action of $X$ on $\Delta$. Hence, if $X$ is transitive on $\Delta$, then $X$ can be embedded into the wreath product of the permutation group induced by the stabiliser $X_{\delta}$ on $\Gamma$ and the permutation group induced by $X$ on $\Delta$. We use this result to describe the case where $X$ is intransitive on $\Delta$ and offer an application to error-correcting codes in Hamming graphs.
\end{abstract}

2010 Mathematics subject classification: primary 05C25; secondary 20B05, 20B25, 20B35, 20D99. Keywords and phrases: wreath products, product action, permutation groups, embedding theorems.

\section{Introduction}

Subgroups of wreath products in product action arise in a number of different contexts. Their importance for group actions is due to the fact that such subgroups give rise to several of the 'O'Nan-Scott types' of finite primitive permutation groups (see [9, Ch. 2] or [8, Sections 1.10 and 4.3]) and finite quasiprimitive groups [13]. They have received special attention recently in the work of Aschbacher [1,2] aimed at studying intervals in subgroup lattices [3] (with Shareshian), and of the authors [4$6,14]$ investigating invariant Cartesian decompositions (with Baddeley). The product

Praeger is supported by Australian Research Council Federation Fellowship FF0776186. Schneider acknowledges the support of the grants PEst-OE/MAT/UI0143/2011 and PTDC/MAT/101993/2008 of the Fundação para a Ciência e a Tecnologia (Portugal) and of the Hungarian Scientific Research Fund (OTKA) grant 72845.

(C) 2012 Australian Mathematical Publishing Association Inc. 1446-7887/2012 \$16.00 
action of the wreath product $W=\operatorname{Sym} \Gamma$ < Sym $\Delta$ is its natural action on the set $\Pi=\operatorname{Func}(\Delta, \Gamma)$ of functions from $\Delta$ to $\Gamma$, described in Section 1.1. If $\Delta=\{1, \ldots, m\}$ then $\Pi$ can be identified with the set $\Gamma^{m}$ of ordered $m$-tuples of elements of $\Gamma$, and in this case subgroups of $W$ arise as automorphism groups of various kinds of graph products, as automorphism groups of codes of length $m$ over the alphabet $\Gamma$ (regarded as subsets of $\Gamma^{m}$ ), and as automorphism groups of a special class of chamber systems in the sense of Tits. To study subgroups $X$ of $W$, and the structures on which they act, one considers the subgroup $H$ of Sym $\Delta$ induced by $X$ along with the 'components' $X^{\Gamma_{\delta}}$, which are permutation groups on $\Gamma$, defined in Section 1.2, for each $\delta \in \Delta$.

We are interested in $X$ up to permutation isomorphism, and wish to replace $X$ by some conjugate in $W$ which gives a simple form with respect to the product action, both for $X$ and the structures on which it acts. This has been done in detail by Kovács [12] in the case where $X$ is primitive on $\Pi$. Kovács also provides a simple form for subgroups inducing a transitive group $H$ on $\Delta$; indeed, his statement [12, (2.2)] is the first assertion of Theorem 1.1(b). One way to handle general subgroups $X$ is to proceed indirectly by appeal to the embedding theorem for subgroups of $W$ using a different action, namely its imprimitive action on $\Gamma \times \Delta$ (see, for example, [7, Theorem 8.5]). However this indirect method does not allow us to keep track of important properties of the underlying product structure. For example, if $X$ is an automorphism group of a code $C \subset \Gamma^{m}$ then we may wish to maintain the property that $C$ contains a specified codeword, say $(\gamma, \ldots, \gamma)$ for a fixed $\gamma \in \Gamma$, as well as to obtain a simple form for the group $X$. Thus a direct approach is highly desirable, and the results of this paper provide such an approach. Our main result shows how to choose a form for $X$ so that the $\delta$-component depends only on the $X$-orbit in $\Delta$ containing $\delta$.

Theorem 1.1. Suppose that $W=\operatorname{Sym} \Gamma$ ? Sym $\Delta$ acts in product action on $\operatorname{Func}(\Delta, \Gamma)$ with base group $B=\operatorname{Func}(\Delta, \operatorname{Sym} \Gamma)$. Let $X \leq W, \varphi \in \operatorname{Func}(\Delta, \Gamma)$ and $\delta_{1} \in \Delta$. Then the following hold.

(a) There is an element $x \in B$ such that the components of $x^{-1} X x$, as defined in (1.4), are constant on each $X$-orbit in $\Delta$. Moreover, if the $\delta$-component of $X$ is transitive on $\Gamma$ for each $\delta \in \Delta$, then the element $x$ can be chosen to fix $\varphi$.

(b) If the group $H$ induced by $X$ on $\Delta$ is transitive, and if $G$ is the $\delta_{1}$-component of $X$, then the element $x$ may be chosen in $\operatorname{Func}(\Delta, \operatorname{Sym} \Gamma)$ such that $X^{x} \leq G \imath H$, (and also such that $\varphi x=\varphi$ if $G$ is transitive on $\Gamma$ ).

Note that, in part (b), $G \succ H$ denotes a particular subgroup of $W$ (defined in Section 1.1) and not just an isomorphism class of groups. If the subgroup $X$ is transitive on $\Pi$ then all of its components are transitive (Theorem 1.2), so the additional condition on the element $x$ in Theorem 1.1 to fix a given point is possible.

Theorem 1.2. Let $W=\operatorname{Sym} \Gamma$ i $\operatorname{Sym} \Delta$ act in product action on $\operatorname{Func}(\Delta, \Gamma)$ with base group $B=\operatorname{Func}(\Delta, \operatorname{Sym} \Gamma)$, where $\Delta, \Gamma$ are finite sets. If $X$ is a transitive subgroup of $W$, then each component of $X$ is transitive on $\Gamma$. Moreover, if $X$ acts transitively on $\Delta$ then each component of the intersection $X \cap B$ is transitive on $\Gamma$. 
In many instances the group $X$ will be far from transitive on $\Pi$, but may still satisfy some transitivity conditions. We give a simple application of Theorem 1.1 in the context of codes. It is most conveniently stated using coordinate notation. So $\Delta=\{1, \ldots, m\}$ and the code $C$ is a subset of $\Gamma^{m}$ with automorphism group being the setwise stabiliser $X$ of $C$ in $W=\operatorname{Sym} \Gamma$ 乙 Sym $\Delta$. The image of $C$ under some element of $W$ is a code equivalent to $C$. Equivalence preserves most important properties, such as the minimum distance $d$ of $C$, which is the minimum number of entries in which distinct elements of $C$ differ. For codes in several interesting families, such as completely transitive codes [11] and neighbour transitive codes [10] with minimum distance at least $3, X$ is transitive on $\Delta$ and all components of $X$ are 2-transitive on $\Gamma$. For such codes with minimum distance $d$, we show that, for our two 'favourite' elements $\gamma, v$ of $\Gamma$, there is a code equivalent to $C$ containing both the $m$-tuple $\left(\gamma^{m}\right)$, and the $m$-tuple $\left(v^{d}, \gamma^{m-d}\right)$ with the first $d$ entries $v$ and the remaining entries $\gamma$, while maintaining a simple form for $X$.

Theorem 1.3. Let $\Delta=\{1, \ldots, m\}$, and let $\gamma, v$ be distinct elements of $\Gamma$. Suppose that $C \subset \Gamma^{m}$ has minimum distance $d$, cardinality $|C|>1$, and automorphism group $X \leq W=\operatorname{Sym} \Gamma$ ? Sym $\Delta$ such that $X$ induces a transitive group $H$ on $\Delta$ and some component $G$ of $X$ is 2-transitive on $\Gamma$. Then there exists $x \in W$ such that the equivalent code $C^{x}$ has automorphism group $X^{x} \leq G<K$ (with $K$ conjugate to $H$ in Sym $\Delta$ ) and $C^{x}$ contains the m-tuples $\left(\gamma^{m}\right)$ and $\left(v^{d}, \gamma^{m-d}\right)$.

1.1. Wreath products and the product action. For our proofs, it is most convenient to use 'function notation' for defining the wreath product and its product action.

Let $\Gamma, \Delta$ be sets and let $G, H$ be subgroups of Sym $\Gamma$, Sym $\Delta$ respectively. Set $B=\operatorname{Func}(\Delta, \mathrm{G})$, the set of functions from $\Delta$ to $G$. Then $B$ is a group with respect to pointwise multiplication of its elements: the product of the functions $f$ and $g$ is the function $f g$ that maps $\delta \mapsto(\delta f)(\delta g)$. Moreover, $B$ is isomorphic to the direct product of $|\Delta|$ copies of $G$ (or the Cartesian product if $\Delta$ is infinite): for $\delta \in \Delta$, set

$$
G_{\delta}=\left\{f \in \operatorname{Func}(\Delta, \mathrm{G}) \mid \delta^{\prime} f=1 \forall \delta^{\prime} \in \Delta \backslash\{\delta\}\right\}
$$

and define the map $\sigma_{\delta}: \operatorname{Func}(\Delta, \mathrm{G}) \rightarrow G_{\delta}$ by

$$
\sigma_{\delta}: f \mapsto f_{\delta} \quad \text { where } \delta^{\prime} f_{\delta}= \begin{cases}\delta f & \text { if } \delta^{\prime}=\delta, \\ 1 & \text { if } \delta^{\prime} \neq \delta\end{cases}
$$

Then $G_{\delta}$ is a subgroup isomorphic to $G, B$ is the direct product of the subgroups $G_{\delta}$ (the Cartesian product if $\Delta$ is infinite), and $\sigma_{\delta}$ is the natural projection map $G \rightarrow G_{\delta}$.

We define a homomorphism $\tau$ from $H$ to Aut $B$ : for $f \in B$ and $h \in H$ let $f(h \tau)$ be the function that maps $\delta \mapsto \delta h^{-1} f$. Now the wreath product $G \imath H$ is defined as the semidirect product $B \rtimes H$ with respect to the homomorphism $\tau$. The normal subgroup $B$ is called the base group of the wreath product, and $H$ is the top group. A useful and easy computation shows that

$$
\left(\delta h^{-1}\right) f=\delta f^{h} \quad \forall h \in H, f \in \operatorname{Func}(\Delta, \mathrm{G}), \delta \in \Delta .
$$


The product action of $G>H$ on $\Pi=\operatorname{Func}(\Delta, \Gamma)$ is defined as follows. Let $f \in$ Func $(\Delta, \mathrm{G}), h \in H$ and set $g=f h$. For $\varphi \in \Pi$ we define $\varphi g$ as the function that maps $\delta \in \Delta$ to

$$
\delta(\varphi g)=\left(\delta h^{-1} \varphi\right)\left(\delta h^{-1} f\right) .
$$

Note that $\delta h^{-1} \varphi \in \Gamma$, and $\delta h^{-1} f \in \operatorname{Sym} \Gamma$. Thus $\left(\delta h^{-1} \varphi\right)\left(\delta h^{-1} f\right) \in \Gamma$, and so $\varphi g \in$ $\operatorname{Func}(\Delta, \Gamma)=\Pi$, as required. It is straightforward to verify that this action of $G<H$ on $\Pi$ is well defined and faithful (see also [9, Section 2.7]).

Let $\gamma$ be a fixed element of $\Gamma$ and let $\varphi$ be the element of $\operatorname{Func}(\Delta, \Gamma)$ that maps $\delta \mapsto \gamma$ for all $\delta \in \Delta$. Let us compute the stabiliser $(G \succ H)_{\varphi}$ of $\varphi$ in $G \imath H$. The subgroup $H \leq(G \imath H)_{\varphi}$ since, if $h \in H$, then $\delta(\varphi h)=\left(\delta h^{-1} \varphi\right)=\gamma$. Therefore $(G \imath H)_{\varphi}=B_{\varphi} H$. Suppose that $f \in B$. Then the image of $\delta \in \Delta$ under $\varphi f$ is

$$
(\delta \varphi)(\delta f)=\gamma(\delta f) .
$$

Hence $f \in B_{\varphi}$ if and only if $\delta f \in G_{\gamma}$ for all $\delta \in \Delta$. Thus

$$
(G \imath H)_{\varphi}=\left\{f h \mid \delta f \in G_{\gamma}, \forall \delta \in \Delta, h \in H\right\}=\operatorname{Func}\left(\Delta, \mathrm{G}_{\gamma}\right) H .
$$

In order to facilitate our discussion of subgroups of wreath products we invoke the language of Cartesian decompositions which was introduced by Baddeley and the authors [4] and was subsequently used to describe innately transitive subgroups of wreath products in product action $[5,6,14]$. Consider the set $\Pi=\operatorname{Func}(\Delta, \Gamma)$, and define, for each $\delta \in \Delta$, a partition $\Gamma_{\delta}$ of $\Pi$ as follows. Set

$$
\Gamma_{\delta}=\left\{\gamma_{\delta} \mid \gamma \in \Gamma\right\} \quad \text { where } \gamma_{\delta}:=\{\varphi \in \Pi \mid \delta \varphi=\gamma\} .
$$

It is routine to check that $\Gamma_{\delta}$ is indeed a partition of $\Pi$. Our notation reflects two important facts. Firstly, the map $\delta \mapsto \Gamma_{\delta}$ is a bijection between $\Delta$ and $\left\{\Gamma_{\delta} \mid \delta \in \Delta\right\}$. Secondly, for a fixed $\delta \in \Delta$, the map $\gamma \mapsto \gamma_{\delta}$ is a bijection between $\Gamma$ and $\Gamma_{\delta}$. For $\gamma \in \Gamma$ and $\delta \in \Delta$, the element $\gamma_{\delta} \in \Gamma_{\delta}$ can be considered as the 'copy' of $\gamma$ in $\Gamma_{\delta}$, and is usually called the $\gamma$-part of $\Gamma_{\delta}$.

The Cartesian product $\prod_{\delta \in \Delta} \Gamma_{\delta}$ can be bijectively identified with the original set $\Pi$. Namely, choosing $\gamma_{\delta} \in \Gamma_{\delta}$, one for each $\delta \in \Delta$, the intersection $\bigcap_{\delta \in \Delta} \gamma_{\delta}$ consists of a single point of $\Pi$, and this gives rise to a bijection from the Cartesian product $\prod_{\delta \in \Delta} \Gamma_{\delta}$ to $\Pi$. Therefore, in the terminology of [4], the set $\left\{\Gamma_{\delta} \mid \delta \in \Delta\right\}$ is called a Cartesian decomposition of $\Pi$. In fact, this set of partitions is viewed as the natural Cartesian decomposition of $\Pi$. As Sym $\Gamma \imath \operatorname{Sym} \Delta$ is a permutation group acting on $\Pi$, the action of Sym $\Gamma$ < Sym $\Delta$ can be extended to subsets of $\Pi$, subsets of subsets, and so on. Hence one can consider the action of Sym $\Gamma$ ? Sym $\Delta$ on the set of partitions of $\Pi$. It is easy to see that $\left\{\Gamma_{\delta} \mid \delta \in \Delta\right\}$ is invariant under this action, and we will see that the (Sym $\Gamma$ < Sym $\Delta$ )-action on this set is permutationally isomorphic to the induced action of Sym $\Gamma$ ? Sym $\Delta$ on $\Delta$ (defined in Section 1.2) under the bijection $\delta \mapsto \Gamma_{\delta}$. The natural product action of Sym $\Gamma$ ? Sym $\Delta$ on $\prod_{\delta \in \Delta} \Gamma_{\delta}$ is permutationally isomorphic to its action on $\Pi$, and indeed the stabiliser in Sym $\Pi$ of this Cartesian decomposition is the wreath product Sym $\Gamma$ ? Sym $\Delta$. See [4] for a more detailed discussion. 
In the case where $\Delta=\{1, \ldots, m\}$, it is worth expressing the product action of the wreath product in coordinate notation. $\operatorname{View} \operatorname{Func}(\Delta, \mathrm{G})$ and $\Pi=\operatorname{Func}(\Delta, \Gamma)$ as $G^{m}$ and $\Gamma^{m}$, respectively. Then, for $\left(\gamma_{1}, \ldots, \gamma_{m}\right) \in \Gamma^{m}$ and $\left(g_{1}, \ldots, g_{m}\right) h \in G \imath H$,

$$
\left(\gamma_{1}, \ldots, \gamma_{m}\right)\left(\left(g_{1}, \ldots, g_{m}\right) h\right)=\left(\gamma_{1 h^{-1}} g_{1 h^{-1}}, \ldots, \gamma_{m h^{-1}} g_{m h^{-1}}\right) .
$$

1.2. Subgroups of wreath products and their components. Suppose that $X \leq$ $\operatorname{Sym} \Gamma$ ? Sym $\Delta$. We define, for $\delta \in \Delta$, the $\delta$-component $X^{\Gamma_{\delta}}$ of $X$ as a subgroup of Sym $\Gamma$ as follows. Recall that each element of $X$ is of the form $f h$, where $f \in \operatorname{Func}(\Delta, \Gamma)$ and $h \in \operatorname{Sym} \Delta$. Recall also the definition of $\Gamma_{\delta}$ in (1.3). Now $X$ permutes the partitions $\Gamma_{\delta}$ and we denote the stabiliser $\left\{x \in X \mid \Gamma_{\delta} x=\Gamma_{\delta}\right\}$ in $X$ of $\Gamma_{\delta}$ by $X_{\Gamma_{\delta}}$. Then $X_{\Gamma_{\delta}}=\{f h \in X \mid \delta h=\delta\}$, and the $\delta$-component $X^{\Gamma_{\delta}}$ of $X$ is the image of $X_{\Gamma_{\delta}}$ in Sym $\Gamma$ under the map $f h \mapsto \delta f$, namely

$$
X^{\Gamma_{\delta}}:=\left\{\delta f \mid \exists f h \in X_{\Gamma_{\delta}} \text { for some } h\right\} .
$$

The bijection $\gamma_{\delta} \mapsto \gamma$ is equivariant with respect to the actions of $X_{\Gamma_{\delta}}$ on $\Gamma_{\delta}$ and $X^{\Gamma_{\delta}}$ on $\Gamma$. Later (when we define the induced action of $X$ on $\Delta$ ) we will see that $\Gamma_{\delta} x=\Gamma_{\delta x}$, for $x \in X$.

In order to prove Proposition 1.2, we need more information about subgroups of $W=\operatorname{Sym} \Gamma \imath \operatorname{Sym} \Delta$ which do not act transitively on $\Delta$. It turns out that such subgroups $X$ may be viewed as subgroups of a direct product in product action: for sets $\Omega_{1}$ and $\Omega_{2}$, and permutation groups $G \leq \operatorname{Sym} \Omega_{1}$ and $H \leq \operatorname{Sym} \Omega_{2}$, the product action of the direct product $G \times H$ is the natural action of $G \times H$ on $\Omega_{1} \times \Omega_{2}$ given by $(g, h):\left(\omega_{1}, \omega_{2}\right) \mapsto\left(\omega_{1} g, \omega_{2} h\right)$ for $\left(\omega_{1}, \omega_{2}\right) \in \Omega_{1} \times \Omega_{2}$ and $(g, h) \in G \times H$. We construct a permutational embedding $(\vartheta, \chi)$ of $X$ acting on $\Pi=\operatorname{Func}(\Delta, \Gamma)$ into Sym $\Omega_{1} \times \operatorname{Sym} \Omega_{2}$ acting on $\Omega_{1} \times \Omega_{2}$, by which we mean a bijection $\chi: \Pi \rightarrow \Omega_{1} \times$ $\Omega_{2}$ and a monomorphism $\vartheta: X \rightarrow \operatorname{Sym} \Omega_{1} \times \operatorname{Sym} \Omega_{2}$ such that, for all $\varphi \in \Pi$ and all $x \in X,(\varphi x) \chi=(\varphi \chi) x \vartheta$.

For a proper nonempty subset $\Delta^{\prime}$ of $\Delta$, and an element $\varphi \in \operatorname{Func}(\Delta, \Gamma)$, define $\left.\varphi\right|_{\Delta^{\prime}} \in \operatorname{Func}\left(\Delta^{\prime}, \Gamma\right)$ as the restriction of $\varphi$ to $\Delta^{\prime}$, so $\left.\delta \varphi\right|_{\Delta^{\prime}}=\delta \varphi$ for all $\delta \in \Delta^{\prime}$. For $X \leq \operatorname{Sym} \Gamma$ ¿ Sym $\Delta$, define the induced action of $X$ on $\Delta$ by $f h: \delta \mapsto \delta h$; equivalently. this is the action $x: \delta \mapsto \delta x$ defined by $\Gamma_{\delta} x=\Gamma_{\delta x}$.

Proposition 1.4. Let $W=\operatorname{Sym} \Gamma$ ¿ Sym $\Delta$, in product action on $\Pi=\operatorname{Func}(\Delta, \Gamma)$, and suppose that $X \leq W$, such that $X$ leaves invariant a proper nonempty subset $\Delta_{0}$ of $\Delta$ in the induced $X$-action on $\Delta$. Let $\Delta_{1}=\Delta \backslash \Delta_{0}$, and set $\Omega_{0}=\operatorname{Func}\left(\Delta_{0}, \Gamma\right)$ and $\Omega_{1}=\operatorname{Func}\left(\Delta_{1}, \Gamma\right)$. Then the following hold .

(a) The map $\vartheta: \Pi \rightarrow \Omega_{0} \times \Omega_{1}$ defined by $\varphi \vartheta=\left(\left.\varphi\right|_{\Delta_{0}},\left.\varphi\right|_{\Delta_{1}}\right)$, for $\varphi \in \Pi$, is a bijection.

(b) The map $\chi: X \rightarrow \operatorname{Sym} \Omega_{0} \times \operatorname{Sym} \Omega_{1}$ defined by $x \chi=\left(x_{0}, x_{1}\right)$, where $\left.\varphi\right|_{\Delta_{i}} x_{i}=$ $\left.(\varphi x)\right|_{\Delta_{i}}$ for $\left.\varphi\right|_{\Delta_{i}} \in \Omega_{i}$, is a monomorphism.

(c) For $i=0,1$, if $\sigma_{i}$ :Sym $\Omega_{0} \times \operatorname{Sym} \Omega_{1} \rightarrow \operatorname{Sym} \Omega_{i}$ is the projection map $\left(x_{0}, x_{1}\right) \sigma_{i}=x_{i}$, then $X \chi \sigma_{i}$ is contained in $W_{i}:=\operatorname{Sym} \Gamma$ i Sym $\Delta_{i}$, and for each $\delta \in \Delta_{i}$, the $\delta$-components of $X$ and $X \chi \sigma_{i}$ are the same subgroup of Sym $\Gamma$. 
(d) $(\vartheta, \chi)$ is a permutational embedding of $X$ on $\Pi$ into the group Sym $\Omega_{0} \times$ Sym $\Omega_{1}$ in its product action on $\Omega_{0} \times \Omega_{1}$, and $X \chi \leq W_{0} \times W_{1}$.

Proof. (a) This follows from the definition of the maps $\left.\varphi\right|_{\Delta_{i}}$ as restrictions of $\varphi$.

(b) Let $x \in X$ and $x \chi=\left(x_{0}, x_{1}\right)$. Note that $\varphi x \in \Pi$, for $\varphi \in \Pi$, and hence $\left.(\varphi x)\right|_{\Delta_{i}} \in \Omega_{i}$. It is straightforward to check that $\left.\left.\varphi\right|_{\Delta_{i}} \mapsto(\varphi x)\right|_{\Delta_{i}}$ is a bijection $\Omega_{i} \rightarrow \Omega_{i}$. Thus $x_{i} \in$ Sym $\Omega_{i}$, for each $i$, and the map $\chi$ is well defined. Let also $y \in X$ and $y \chi=\left(y_{0}, y_{1}\right)$. It follows immediately from the definition of the $x_{i}$ and $y_{i}$ that $(x y)_{i}=x_{i} y_{i}$ for each $i$, and hence that $x \chi y \chi=(x y) \chi$. Thus $\chi$ is a homomorphism. If $x \in \operatorname{ker} \chi$ then, for each $\varphi \in \Pi$ and each $i,\left.\varphi\right|_{\Delta_{i}}=\left.\varphi\right|_{\Delta_{i}} x_{i}=\left.(\varphi x)\right|_{\Delta_{i}}$. Thus $\varphi=\varphi x$. Since this holds for all $\varphi \in \Pi, x=1$.

(c) As in (1.3), for each $\delta \in \Delta_{i}$ we define a partition $\Gamma_{\delta}^{i}$ of $\Omega_{i}$ as follows. For $\gamma \in \Gamma$, we define $\gamma_{\delta}^{i}=\left\{\psi \in \Omega_{i} \mid \delta \psi=\gamma\right\}$ and $\Gamma_{\delta}^{i}=\left\{\gamma_{\delta}^{i} \mid \gamma \in \Gamma\right\}$. Since $\left.(\varphi x)\right|_{\Delta_{i}}=\varphi_{\Delta_{i}} x_{i}$ we have $\gamma_{\delta}^{i} x_{i}=\gamma_{\delta x}^{i}$ so that $\Gamma_{\delta}^{i} x_{i}=\Gamma_{\delta x}^{i}$. Thus $X \chi \sigma_{i}$ leaves invariant the set of partitions $\left\{\Gamma_{\delta}^{i} \mid \delta \in \Delta_{i}\right\}$ which forms a Cartesian decomposition of $\Omega_{i}$. Hence $X \chi \sigma_{i}$ is contained in $W_{i}$. The stabiliser of $\Gamma_{\delta}^{i}$ in $X \chi \sigma_{i}$ is $\left(X_{\Gamma_{\delta}}\right) \chi \sigma_{i}$ and the $\delta$-component of $X \chi \sigma_{i}$, defined as in (1.4), is equal to the $\delta$-component $X^{\Gamma_{\delta}}$ of $X$.

(d) This follows since, for all $\varphi \in \Pi$ and all $x \in X$,

$$
\varphi \vartheta x \chi=\left(\left.\varphi\right|_{\Delta_{0}},\left.\varphi\right|_{\Delta_{1}}\right) x \chi=\left(\left.(\varphi x)\right|_{\Delta_{0}},\left.(\varphi x)\right|_{\Delta_{1}}\right)=(\varphi x) \vartheta
$$

and since, by part (c), $X \chi \sigma_{i} \leq W_{i}$.

\section{Proof of Theorem 1.1}

Suppose that $W=\operatorname{Sym} \Gamma$ ? Sym $\Delta$ acts in product action on $\Pi=\operatorname{Func}(\Delta, \Gamma)$ with base group $B=\operatorname{Func}(\Delta, \operatorname{Sym} \Gamma)$. Let $X \leq W, \varphi \in \operatorname{Func}(\Delta, \Gamma)$ and $\delta_{1} \in \Delta$. Note that $B$ is the kernel of the induced action of $W$ on $\Delta$, so if $x \in B$, then the $X$-orbits in $\Delta$ coincide with the $x^{-1} X x$-orbits in $\Delta$. For the computations in the proof we often use the properties given in (1.1) and (1.2), and the equality $\delta\left(f f^{\prime}\right)=(\delta f)\left(\delta f^{\prime}\right)$, for $f, f^{\prime} \in B$, $h \in \operatorname{Sym} \Delta, \delta \in \Delta$.

Let $\Delta_{1}, \ldots, \Delta_{r}$ be the $X$-orbits in $\Delta$ under the action induced by $X$ on $\Delta$. For $1 \leq i \leq r$ choose $\delta_{i} \in \Delta_{i}$, with $\delta_{1}$ as in the previous paragraph if $i=1$. For each $\delta \in \Delta_{i}$, choose $t_{\delta} \in X$ such that $\Gamma_{\delta_{i}} t_{\delta}=\Gamma_{\delta}$, and in particular take $t_{\delta_{i}}=1$. Then $t_{\delta}=f_{\delta} h_{\delta}$ with $f_{\delta} \in B$ and $h_{\delta} \in$ Sym $\Delta$ such that $\delta_{i} h_{\delta}=\delta$. Also $X_{\Gamma_{\delta}}=\left(X_{\Gamma_{\delta_{i}}}\right)^{t_{\delta}}$.

Claim 1. If the $\delta_{i}$-component is transitive on $\Gamma$, then we may assume in addition that, for each $\delta \in \Delta_{i}, \delta_{i} f_{\delta}$ fixes the point $\delta \varphi$ of $\Gamma$.

Since we have $t_{\delta_{i}}=1$, the element $\delta_{i} f_{\delta_{i}}$ is the identity of Sym $\Gamma$ and hence fixes $\delta \varphi$. Let $\delta \in \Delta_{i} \backslash\left\{\delta_{i}\right\}$ and consider $s_{\delta}=f h \in X_{\Gamma_{\delta_{i}}}$ with $f \in B$ and $h \in \operatorname{Sym} \Delta$. Then $\delta_{i} h=\delta_{i}$, and the element $s_{\delta} t_{\delta}$ is equal to $f_{\delta}^{\prime} h_{\delta}^{\prime}$, with $f_{\delta}^{\prime}=f f_{\delta}^{h^{-1}}$ and $h_{\delta}^{\prime}=h h_{\delta}$, and satisfies $\Gamma_{\delta_{i}} s_{\delta} t_{\delta}=\Gamma_{\delta}$. Moreover, $\delta_{i} f_{\delta}^{\prime}=\left(\delta_{i} f\right)\left(\left(\delta_{i} h\right) f_{\delta}\right)=\left(\delta_{i} f\right)\left(\delta_{i} f_{\delta}\right)$, and we note that $\delta_{i} f \in \operatorname{Sym} \Gamma$ lies in the $\delta_{i}$-component of $X$; see (1.4). If the $\delta_{i}$-component is transitive on $\Gamma$, then we may choose $s_{\delta}$ in $X_{\Gamma_{\delta_{i}}}$ such that the element $\left(\delta_{i} f\right)\left(\left(\delta_{i}\right) f_{\delta}\right)$ fixes $\delta \varphi$. Replacing $t_{\delta}$ by $s_{\delta} t_{\delta}$ gives an element with the required properties. 
Claim 2. For $\delta \in \Delta_{i}$, the $\delta$-component $X^{\Gamma_{\delta}}$ equals $\left(X^{\Gamma_{\delta_{i}}}\right)^{\delta_{i} f_{\delta}}$.

Let $\delta_{i} f \in X^{\Gamma_{\delta_{i}}}$. By (1.4), there exists $h \in \operatorname{Sym} \Delta$ such that $\delta_{i} h=\delta_{i}$ and $f h \in X_{\Gamma_{\delta_{i}}}$. Therefore $X_{\Gamma_{\delta}}$ contains

$$
(f h)^{t_{\delta}}=f^{t_{\delta}} h^{f_{\delta} h_{\delta}}=f^{t_{\delta}}\left(f_{\delta}^{-1} f_{\delta}^{h^{-1}} h\right)^{h_{\delta}}=f^{t_{\delta}}\left(f_{\delta}^{-1}\right)^{h_{\delta}} f_{\delta}^{h^{-1} h_{\delta}} h^{h_{\delta}} .
$$

This implies that the $\delta$-component $X^{\Gamma_{\delta}}$ contains

$$
\delta\left(f^{t_{\delta}}\left(f_{\delta}^{-1}\right)^{h_{\delta}} f_{\delta}^{h^{-1} h_{\delta}}\right)=\left(\left(\delta h_{\delta}^{-1}\right) f^{f_{\delta}}\right)\left(\left(\delta h_{\delta}^{-1}\right) f_{\delta}^{-1}\right)\left(\left(\delta h_{\delta}^{-1} h\right) f_{\delta}\right)
$$

and using the facts that $\delta_{i} h_{\delta}=\delta$ and $\delta_{i} h=\delta_{i}$, this is equal to

$$
\left(\left(\delta_{i}\right) f^{f_{\delta}}\right)\left(\left(\delta_{i}\right) f_{\delta}^{-1}\right)\left(\left(\delta_{i}\right) f_{\delta}\right)=\delta_{i}\left(f^{f_{\delta}} f_{\delta}^{-1} f_{\delta}\right)=\delta_{i} f^{f_{\delta}}=\left(\delta_{i} f\right)^{\delta_{i} f_{\delta}} .
$$

Thus $X^{\Gamma_{\delta}}$ contains $\left(X^{\Gamma_{i}}\right)^{\delta_{i} f_{\delta}}$, and a similar argument proves the reverse inclusion. Hence equality holds and the claim is proved.

Definition of $x$ : Define $x \in B=\operatorname{Func}(\Delta, \operatorname{Sym} \Gamma)$ as the function satisfying, for each $i$ and each $\delta \in \Delta_{i}, \delta x=\delta_{i} f_{\delta}^{-1}$. If all components of $X$ are transitive on $\Gamma$ then we assume (as we may by Claim 1) in addition that, for each $i$ and $\delta \in \Delta_{i}, \delta_{i} f_{\delta}$ fixes the point $\delta \varphi$, and hence $\delta x=\delta_{i} f_{\delta}^{-1}=\left(\delta_{i} f_{\delta}\right)^{-1}$ fixes $\delta \varphi$. Thus in this case $x$ fixes $\varphi$.

Claim 3. The components of $x^{-1} X x$ are constant on each of the $\Delta_{i}$.

Since $x$ acts trivially on $\Delta$, the stabiliser $\left(X^{x}\right)_{\Gamma_{\delta}}=\left(X_{\Gamma_{\delta}}\right)^{x}$ for each $\delta \in \Delta$. Thus $\delta f$ lies in the $\delta$-component $X^{\Gamma_{\delta}}$ if and only if there exists $h \in \operatorname{Sym} \Delta$ such that $f h \in X_{\Gamma_{\delta}}$ or, equivalently, $(f h)^{x}=f^{x} x^{-1} x^{h^{-1}} h \in\left(X^{x}\right)_{\Gamma_{\delta}}$. This implies that the $\delta$-component of $X^{x}$ contains

$$
\delta\left(f^{x} x^{-1} x^{h^{-1}}\right)=\delta\left(x^{-1} f x^{h^{-1}}\right)=\left(\delta x^{-1}\right)(\delta f)((\delta h) x)=(\delta f)^{\delta x}
$$

since $\delta h=\delta$. Thus the $\delta$-component of $X^{x}$ contains $\left(X^{\Gamma_{\delta}}\right)^{\delta x}$ and a similar argument proves the reverse inclusion, so equality holds. Now $\delta x=\delta_{i} f_{\delta}^{-1}=\left(\delta_{i} f_{\delta}\right)^{-1}$, which by Claim 2 conjugates $X^{\Gamma_{\delta}}$ to $X^{\Gamma_{\delta_{i}}}$. Thus

$$
\left(X^{x}\right)^{\Gamma_{\delta}}=\left(X^{\Gamma_{\delta}}\right)^{\delta x}=\left(X^{\Gamma_{\delta}}\right)^{\delta_{i} f_{\delta}^{-1}}=X^{\Gamma_{\delta_{i}}}
$$

for all $\delta \in \Delta_{i}$. This completes the proof of Claim 3, and part (a) follows.

To prove part (b) we assume that the group $H$ induced by $X$ on $\Delta$ is transitive, and let $G$ be the $\delta_{1}$-component of $X$. From what we have just proved, each component of $X^{x}$ is equal to $G$. Let $g^{\prime}$ be an arbitrary element of $X^{x}$. Then $g^{\prime}=x^{-1} g x$ for some $g \in X$, and we have $g=f h$ with $f \in B$ and $h \in \operatorname{Sym} \Delta$. By the definition of $H$, we have $h \in H$. Also

$$
g^{\prime}=x^{-1} f h x=\left(x^{-1} f x^{h^{-1}}\right) h=f^{\prime} h, \quad \text { say. }
$$

Thus, in order to prove that $g^{\prime} \in G \imath H$, it is sufficient to prove that, for each $\delta \in \Delta$, $\delta f^{\prime} \in G$. 
Let $\delta^{\prime}:=\delta h$. Then $h h_{\delta^{\prime}}^{-1} h_{\delta}$ fixes $\delta$, and so $X_{\Gamma_{\delta}}$ contains

$$
g t_{\delta^{\prime}}^{-1} t_{\delta}=f h h_{\delta^{\prime}}^{-1} f_{\delta^{\prime}}^{-1} f_{\delta} h_{\delta}=f\left(f_{\delta^{\prime}}^{-1} f_{\delta}\right)^{h_{\delta^{\prime}} h^{-1}} h h_{\delta^{\prime}}^{-1} h_{\delta} .
$$

Hence $\left(X^{x}\right)_{\Gamma_{\delta}}=\left(X_{\Gamma_{\delta}}\right)^{x}$ contains

$$
x^{-1} g t_{\delta^{\prime}}^{-1} t_{\delta} x=\left(x^{-1} f\left(f_{\delta^{\prime}}^{-1} f_{\delta}\right)^{h_{\delta^{\prime}} h^{-1}} x^{h_{\delta}^{-1} h_{\delta^{\prime}} h^{-1}}\right) h h_{\delta^{\prime}}^{-1} h_{\delta}
$$

which equals $f^{\prime \prime} h h_{\delta^{\prime}}^{-1} h_{\delta}$, say. This means that the $\delta$-component $G$ of $X^{x}$ contains

$$
\begin{aligned}
\delta f^{\prime \prime} & =\left(\delta x^{-1} f\right)\left(\left(\delta h h_{\delta^{\prime}}^{-1}\right)\left(f_{\delta^{\prime}}^{-1} f_{\delta}\right)\right)\left(\left(\delta h h_{\delta^{\prime}}^{-1} h_{\delta}\right) x\right) \\
& =\left(\delta x^{-1} f\right)\left(\delta_{1}\left(f_{\delta^{\prime}}^{-1} f_{\delta}\right)\right)(\delta x) .
\end{aligned}
$$

By the definition of $x, \delta_{1}\left(f_{\delta^{\prime}}^{-1} f_{\delta}\right)=\left(\delta_{1} f_{\delta^{\prime}}^{-1}\right)\left(\delta_{1} f_{\delta}\right)=\left(\delta^{\prime} x\right)(\delta x)^{-1}$. It follows that

$$
\delta f^{\prime \prime}=\left(\delta x^{-1} f\right)\left(\delta^{\prime} x\right)=\left(\delta x^{-1} f\right)\left(\delta x^{h^{-1}}\right)=\delta\left(x^{-1} f x^{h^{-1}}\right)=\delta f^{\prime} .
$$

Therefore $\delta f^{\prime} \in G$, as required. Thus part (b) is proved, completing the proof of Theorem 1.1.

\section{Proof of Theorem 1.2}

Let $W=\operatorname{Sym} \Gamma$ isym $\Delta$ act in product action on $\Pi=\operatorname{Func}(\Delta, \Gamma)$ with base group $B=\operatorname{Func}(\Delta, \operatorname{Sym} \Gamma)$, where $\Delta, \Gamma$ are finite sets. Suppose that $X$ is a transitive subgroup of $W$, and let $K:=X \cap B$.

Let $\delta \in \Delta$, let $\Delta_{0}$ be the orbit of $X$ in $\Delta$ containing $\delta$, and let $\Delta_{1}=\Delta \backslash \Delta_{0}$. By Proposition 1.4(d), the permutation actions of $X$ on $\Pi$ and on $\Omega_{0} \times \Omega_{1}$ are equivalent. In particular, as $X$ is transitive on $\Pi$, its projection $X \sigma_{0}$ is transitive on $\Omega_{0}$. Further (defining $\Gamma_{\delta}^{0}$ as in the proof of Proposition 1.4(c)), if $X^{\Gamma_{\delta}^{0}}$ is transitive then, by Proposition 1.4(c), $X^{\Gamma_{\delta}}$ is transitive. Thus it suffices to prove that all components of $X$ are transitive in the case where $X$ acts transitively on $\Delta$. So assume that $X$ is transitive on $\Delta$. Let $r:=|\Delta|$, and suppose that, for some $\delta \in \Delta$, the $\delta$-component $X^{\Gamma_{\delta}}$ is intransitive. Now $K$ is a normal subgroup of $X_{\Gamma_{\delta}}$ and hence, by (1.4), the $\delta$-component $K^{\Gamma_{\delta}}$ of $K$ is a normal subgroup of $X^{\Gamma_{\delta}}$. Hence $K^{\Gamma_{\delta}}$ has $s$ orbits in its action on $\Gamma$ for some $s>1$. Since $X$ is transitive on $\Delta$ and normalises $K$, it follows that $K^{\Gamma_{\delta}}$ has $s$ orbits for each $\delta \in \Delta$. Define $L:=\left\{f \in B \mid \delta f \in K^{\delta}\right.$ for each $\left.\delta \in \Delta\right\}$. Then $L \cong \prod_{\delta \in \Delta} K^{\delta}, L$ has $s^{r}$ orbits in $\Pi$, and $K \leq L \cap X$. Moreover, $X$ normalises $L$ and, since $X$ is transitive on $\Pi$, it permutes the $s^{r}$ orbits of $L$ transitively and $K$ lies in the kernel of this action. Thus $|X / K|$ is divisible by $s^{r}$. However $X / K$ is isomorphic to the transitive group induced by $X$ on $\Delta$ and hence $|X / K|$ divides $r$ !. Thus $s^{r}$ divides $r$ !. However, this is impossible since for any prime $p$ dividing $s$, the order of a Sylow $p$-subgroup of Sym $\Delta$ is at most $p^{r-1}$. Thus $s=1$. This proves both assertions of Theorem 1.2. 


\section{Proof of Theorem 1.3}

Let $\Delta=\{1, \ldots, m\}$, and let $\gamma, v$ be distinct elements of $\Gamma$. Suppose that $C \subset \Gamma^{m}$ has minimum distance $d$, cardinality $|C|>1$, and automorphism group $X \leq W=\operatorname{Sym} \Gamma$ ? Sym $\Delta$ such that $X$ induces a transitive group $H$ on $\Delta$ and the 1-component $X^{\Gamma_{1}}$ of $X$ is a 2-transitive subgroup $G$ of Sym $\Gamma$. In this context it is convenient to identify $\Pi=\operatorname{Func}(\Delta, \Gamma)$ with $\Gamma^{m}$, and the base group $B$ of $W$ with $(\operatorname{Sym} \Gamma)^{m}$. Under this identification, for example, the subgroup $L=\left\{f \in B \mid \delta f \in X^{\Gamma_{\delta}}\right.$ for all $\left.\delta \in \Delta\right\}$ of $B$ is identified with the direct product $\prod_{\delta \in \Delta} X^{\Gamma_{\delta}}$ of the components of $X$. Moreover, since $X$ acts transitively on $\Delta$, each of the $X^{\Gamma_{\delta}}$ is 2-transitive on $\Gamma$.

Let $a:=\left(\gamma_{1}, \ldots, \gamma_{m}\right), b:=\left(\beta_{1}, \ldots, \beta_{m}\right) \in C$ be codewords at distance $d$. Since $G$ is transitive on $\Gamma$, the subgroup $L$ of the base group $B$ is transitive on $\Gamma^{m}$, so there is an element $x_{1} \in L$ such that $a x_{1}=\left(\gamma^{m}\right)$. Then, since $x_{1}$ normalises each of the direct factors $X^{\Gamma_{\delta}}$ of $L$, it follows that $X^{x_{1}}$ has the same components as $X$. Now we apply Theorem 1.1(b) and obtain an element $x_{2} \in B$ such that $X^{x_{1} x_{2}} \leq G \succ H$ and $a x_{1} x_{2}=\left(\gamma^{m}\right) x_{2}=\left(\gamma^{m}\right)$. Now the image $b x_{1} x_{2}$ differs from $\left(\gamma^{m}\right)$ in exactly $d$ entries. Let $I$ denote this $d$-subset of $\Delta$. Choose $x_{3}$ in the top group Sym $\Delta$ of $W$ such that $I x_{3}=\{1, \ldots, d\}$. Then $C x_{1} x_{2} x_{3}$ contains $a x_{1} x_{2} x_{3}=\left(\gamma^{m}\right) x_{3}=\left(\gamma^{m}\right)$ and $b x_{1} x_{2} x_{3}$, and the latter $m$-tuple differs from $\left(\gamma^{m}\right)$ precisely in the $d$-subset $I x_{3}=\{1, \ldots, d\}$. Thus entries $d+1, \ldots, m$ of $b x_{1} x_{2} x_{3}$ are all equal to $\gamma$. The automorphism group $X^{x_{1} x_{2} x_{3}}$ of $C x_{1} x_{2} x_{3}$ has the same components as $X^{x_{1} x_{2}}$ (which are all equal to $G$ ) and induces the transitive group $K:=H^{x_{2}}$ on $\Delta$. Thus $X^{x_{1} x_{2} x_{3}} \leq G \succ K$. Finally, since $G$ is 2-transitive on $\Gamma$, for each $i \leq d$ there is an element $y_{i} \in G_{\gamma}$ which maps the $i$ th entry of $b x_{1} x_{2} x_{3}$ to $v$. Let $x_{4} \in \operatorname{Func}\left(\Delta, \mathrm{G}_{\gamma}\right) \leq B$ be any element such that $i x_{4}=y_{i}$ for $i=1, \ldots, d$, and set $x=x_{1} x_{2} x_{3} x_{4}$. Then $X^{x} \leq G \imath K$ and $C x$ contains $\left(\gamma^{m}\right)$ and $\left(v^{d}, \gamma^{m-d}\right)$.

\section{Acknowledgement}

The authors are grateful to an anonymous referee for suggestions leading to clarification and improvement of the exposition.

\section{References}

[1] M. Aschbacher, 'Overgroups of primitive groups', J. Aust. Math. Soc. 87 (2009), 37-82.

[2] M. Aschbacher, 'Overgroups of primitive groups. II', J. Algebra 322 (2009), 1586-1626.

[3] M. Aschbacher and J. Shareshian, 'Restrictions on the structure of subgroup lattices of finite alternating and symmetric groups', J. Algebra 322 (2009), 2449-2463.

[4] R. W. Baddeley, C. E. Praeger and C. Schneider, 'Transitive simple subgroups of wreath products in product action', J. Aust. Math. Soc. 77 (2004), 55-72.

[5] R. W. Baddeley, C. E. Praeger and C. Schneider, 'Innately transitive subgroups of wreath products in product action', Trans. Amer. Math. Soc. 358 (2006), 1619-1641.

[6] R. W. Baddeley, C. E. Praeger and C. Schneider, 'Intransitive Cartesian decompositions preserved by innately transitive permutation groups', Trans. Amer. Math. Soc. 360 (2008), 743-764.

[7] M. Bhattacharjee, D. Macpherson, R. G. Möller and P. M. Neumann, Notes on Infinite Permutation Groups, Texts and Readings in Mathematics, 12 (Hindustan Book Agency, New Delhi, 1997; copublished by Springer, Berlin, 1997). 
[8] P. J. Cameron, Permutation Groups, London Mathematical Society Student Texts, 45 (Cambridge University Press, Cambridge, 1999).

[9] J. D. Dixon and B. Mortimer, Permutation Groups, Graduate Texts in Mathematics, 163 (Springer, New York, 1996).

[10] N. Gillespie, 'Neighbour transitive codes', PhD Thesis, The University of Western Australia, 2011.

[11] M. Giudici and C. E. Praeger, 'Completely transitive codes in Hamming graphs', European J. Combin. 20 (1999), 647-661.

[12] L. G. Kovács, 'Wreath decompositions of finite permutation groups', Bull. Aust. Math. Soc. 40 (1989), 255-279.

[13] C. E. Praeger, 'An O'Nan-Scott theorem for finite quasiprimitive permutation groups and an application to 2-arc transitive graphs', J. Lond. Math. Soc. (2) 47 (1993), 227-239.

[14] C. E. Praeger and C. Schneider, 'Three types of inclusions of innately transitive permutation groups into wreath products in product action', Israel J. Math. 158 (2007), 65-104.

CHERYL E. PRAEGER, Centre for Mathematics of Symmetry and Computation, School of Mathematics and Statistics, The University of Western Australia, 35 Stirling Highway, Crawley, Western Australia 6009, Australia e-mail: cheryl.praeger@uwa.edu.au

CSABA SCHNEIDER, Centro de Álgebra da Universidade de Lisboa, Av. Prof. Gama Pinto, 2, 1649-003 Lisboa, Portugal

e-mail: csaba.schneider@gmail.com 